\title{
FACTORES QUE INTERVIENEN EN LA UBICACIÓN LABORAL DE LOS EGRESADOS DE LA UNAD ECACEN ZOOC
}

\section{FACTORS INVOLVED IN THE EMPLOYMENT LOCATION OF UNAD ECACEN ZOOC GRADUATES}

Autor 1 - Nelson Augusto Serna Porras

Maestrando en Administración de Organizaciones, Administrador de Empresas. Docente ocasional de la UNAD, y perteneciente al grupo de investigación CANANGUCHALES (Código COL0060156), y al semillero de investigación SIDESGOP.

ORCID 0000-0002-4575-4645

email.nelson.serna@unad.edu.co

Autor 2 - Guillermo Giraldo Vargas

Administrador de empresas, especialista en gestión de costos, magister en administración de organizaciones y tecnólogo en gestión agropecuaria, docente ocasional de la UNAD y perteneciente al grupo de investigación CANANGUCHALES (Código COL0060156), y al semillero de investigación SIDESGOP.

ORCID 0000-0003-2695-4745

email.guillermo.giraldo@unad.edu.co

\section{RESUMEN}

El acceso al empleo constituye uno de los factores que ocupan en mayor mediada a las diferentes naciones debido al desajuste existente entre la demanda laboral y la oferta de personal competente, asunto que se relaciona directamente con la productividad de los países y que preocupa a cada individuo que necesita generar ingresos para su subsistencia, hecho que impacta en la población de los recién egresados de las Instituciones de Educación Superior (IES), que están sometidos a las difíciles exigencias de en un mundo laboral altamente competido, dado que su falta de experiencia profesional constituye un detractor al momento ser elegido en $\mathrm{n}$ procesos de selección, este asunto forma parte del presente estudio de carácter cuantitativo en el cual se ha consultado información mediante la aplicación de una encuesta. La investigación de tipo cuantitativo y carácter exploratorio buscó conocer las opiniones de los egresados sobre aspectos presentes al buscar empleo, que tienen que ver, en parte, con las gestiones de la universidad y con la disposición del sector productivo para contratar recién egresados de la Universidad Nacional Abierta y a Distancia (UNAD) en la Escuela de Ciencias Administrativas, Contables, Económicas y de Negocios (ECACEN) de la Zona Occidente (ZOCC); el instrumento fue aplicado en el 
mes de noviembre de 2020 a una población de 1164 egresados de la ZOCC con una muestra de 289 de los graduados y se obtuvo un total de 324 respuestas, también se buscó información en fuentes secundarias. Los resultados logrados permiten observar como un gran porcentaje de los egresados considera que su paso por la universidad ayudó a desarrollar competencias aplicables en el mercado laboral, adicionalmente, al consultar sobre los principales factores presentes en el proceso de búsqueda de empleo, se pudo ver que sobresalen palabras como: experiencia, formación, competencias, conocimiento y oportunidades; la falta de respuesta a otras preguntas dejan la sensación de una posible lejanía o desinterés de los egresados hacia la UNAD ECACEN ZOCC, además se puede mencionar sobre los datos recabados que, se evidencia un desinterés de los encuestados en relación con la necesidad de aprender a gestionar bolsas de empleo al momento de buscar una ubicación laboral; lo cual puede ser usado como material para nuevas investigaciones orientadas a lograr una relación mas cercana entre los graduados y la institución.

\section{PALABRAS CLAVE}

Factores; mercado laboral; educación superior; oferta de estudiantes; demanda laboral.

\section{ABSTRACT}

Access to employment is one of the factors that occupies the different nations to a greater extent due to the mismatch between labor demand and the supply of competent personnel, a matter that is directly related to the productivity of the countries and that concerns each individual that needs to generate income for their subsistence, a fact that impacts on the population of recent graduates from Higher Education Institutions (HEIs), who are subjected to the difficult demands of a highly competitive world of work, given that their lack of professional experience constitutes a detractor at the time of being chosen in a selection process, this matter is part of this quantitative study in which information has been consulted through the application of a survey. The research of a quantitative and exploratory nature sought to know the opinions of graduates on aspects present when seeking employment, which have to do, in part, with the efforts of the university and with the willingness of the productive sector to hire recent graduates of the University National Open and Distance (UNAD) at the School of Administrative, Accounting, Economic and Business Sciences (ECACEN) of the Western Zone (ZOCC); The instrument was applied in November 2020 to a population of 1,164 ZOCC graduates with a sample of 289 graduates, and a total of 324 responses were obtained.

\section{KEYWORDS}

Factors; working market; higher education; offer of students; labor demand. 


\section{INTRODUCCIÓN}

En la actualidad el mercado laboral se constituye como un escenario complejo dada la baja oferta de empleos en relación con la cantidad de profesionales que requieren un trabajo (Rus \& Gragmena, 2018), esta situación se agrava si se considera el panorama de sobre educación presente en los últimos años en los países que se encuentran en proceso de desarrollo (Nina \& Eliana, 2019) y que además presentan desventajas en relación con digitalización que ha transformado de manera profunda las formas productivas y de generación de nuevas formas de empleo (Arall \& Molías, 2019), además, conseguir un trabajo depende en gran medida de la calidad de la educación impartida desde las IES (González Jaimes \& Miguel Silva, 2017); se trata de un asunto de precariedad laboral que no solo se presenta en Colombia sino en los demás países de la región (Fern\&aacute et al., 2015) el cual puede ser enfrentado mediante el desarrollo de estrategias que permitan fortalecer la relación entre las universidades, los egresados y el sector productivo.

La UNAD no es ajena a esta situación que también afecta a sus estudiantes, incluidos los matriculados en los programas que se ofertan en la ECACEN de la ZOCC, por ello se realizó el presente estudio con el fin de conocer lo que opinan los egresados sobe los aspectos al momento de buscar una ubicación laboral, sobre la gestión de la universidad para apoyar este proceso y sobre la disposición del sector productivo para contratar recién egresados, lo cual ha dado paso a la siguiente pregunta de investigación: ¿Cuáles son los factores que intervienen en la ubicación laboral de los egresados de la ECACEN ZOOC?

\section{METODOLOGÍA}

La presente investigación se aborda desde un enfoque cuantitativo de corte exploratorio con el fin de conocer sobre el contexto de la investigación o problema de estudio, y sus principales características, utilizando para ello la organización de la información a través de tablas de frecuencia que permiten observar la medición de variables y gráficas explicativas, de tal manera que los datos resultantes de la aplicación de la encuesta y la información secundaría obtenida de revisiones bibliográficas se pueda interpretar mejor e inferir de acuerdo a sus resultados; se ha buscado tener conocimiento de los factores que los egresados mencionan alrededor del tema de la búsqueda de empleo y así generar conclusiones. En cuanto a la información primaria, se diseñó y aplicó una encuesta en el mes de noviembre de 2020 a una población " $\mathrm{N}$ " de 1164 egresados de la ZOCC, contemplando un error " $\mathrm{E}$ " del $5 \%$, una probabilidad de éxito " $p$ " del $50 \%$ y una probabilidad de fracaso " $q$ " de igual porcentaje, además, un nivel de confianza " $Z$ " del 95\%, lo cual 
permitió determinar una muestra de 289 de los graduados; sin embargo, se obtuvo un total de 324 respuestas.

\section{REVISIÓN DOCUMENTAL}

En Colombia la tasa de desempleo para el mes de septiembre de 2019 volvió a ser de dos dígitos $(10,2 \%)$, en comparación con el mismo mes del año anterior que fue del 9,5\%, lo que indica un incremento del 0,7\% (Banco de la Republica, 2019), hecho que no resulta alentador para una población creciente que cuenta con un total de 48.258 .494 habitantes según el censo realizado en 2018 (DANE, 2018), de los cuales el 68,2\% se encuentra en un rango de edad comprendido entre los 15 y 65 años que corresponde a una porción de la población en edad de trabajar que demanda una mayor cantidad de servicios de toda índole. Adicionalmente, los efectos generados por la pandemia del COVID-19 han dejado una tasa del 17,3\% en enero de 2021 (Banco de la Republica, 2019), lo que representa un alto crecimiento del desempleo para una población económicamente afectada por la pandemia.

El anterior panorama del mercado laboral es objeto de análisis debido a que, además de los efectos del Coronavirus, puede deberse a aspectos fundamentales de la economía, como la disminución de la demanda de bienes y servicios, el contrabando de bienes, el cambio en los ciclos económicos o la migración de personas hacia Colombia, como es el caso actual con la población venezolana; también puede deberse a otros aspectos como la gestión de las IES, los prejuicios de los responsables de contratación en las empresas, entre muchos otros.

Desde un punto de vista macroeconómico, el empleo es determinante para el desarrollo de la economía de cada país, debido a que es uno de los tres elementos que se relacionan en el "ciclo económico" junto con la inflación y el crecimiento; cuyo comportamiento determina la "expansión (recuperación)" o "contracción (recesión)" de la economía (Dornbusch, Fisher y Startz, 2009). En la medida que haya una relación más estrecha entre la oferta de egresados de las IES y la demanda laboral, la dinámica de empleo puede ser más rápida y eficiente, contribuyendo así al logro de un mayor índice de mano de obra empleada que, en todo caso, nunca logrará alcanzar el nivel de "pleno empleo" según el cual "todos los que buscan trabajo lo encuentran en un tiempo razonable" (Fischer et al., 1985). Independientemente de que el empleo sea formal o informal, representa un tema delicado al cual los países le dedican mucha atención, no solo porque de este depende en parte su productividad y desarrollo, sino también porque:

En la actualidad el mercado laboral se encuentra en un escenario de constantes cambios y vertiginosas transformaciones, originadas por 
las crisis económicas, el crecimiento acelerado de la población económicamente activa, el incremento de la incorporación de la mujer al trabajo, el desarrollo diversificado en varias regiones, cambios demográficos y flexibilización de las relaciones laborales, esto ha provocado un entorno laboral cada vez más diversificado, especializado y globalizado, en el que la precariedad y el desempleo se consolidan como características importantes, (Romero Fernández \& Álvarez Gavilanes, 2015).

Desde el punto de vista laboral, los diferentes intermediarios del mercado de trabajo tales como empresas temporales y bolsas de empleo, las empresas públicas y privadas, las IES y la población misma constituyen algunos de los elementos más importantes que interactúan en la dinámica del empleo, que es compleja por su múltiples variables y por las fuerzas que intervienen en su evolución, esta interacción da paso a lo que se conoce como la fluidez del mercado laboral y de empleo los cuales, según (Morales \& Medina (2019) corresponden a "la movilidad de trabajadores y empleos en el mercado laboral". La cual, desde luego, influye en la posibilidad de colocación de los egresados de las diferentes IES para quienes la problemática laboral no se concentra únicamente en la posibilidad de emplearse sino también de la calidad de las condiciones laborales que se ofertan para ellos.

Frente a lo discutido hasta el momento surge la necesidad de encontrar o crear herramientas que ayuden a la construcción de procesos de apoyo para una adecuada inserción laboral de los egresados universitarios, esto depende en gran medida del entendimiento que se tenga sobre los factores que intervienen en la relación existente entre la oferta de egresados de las IES, el sector productivo y los egresados, se buscó entonces conocer las opiniones de los egresados sobre aspectos presentes al buscar empleo, que tienen que ver, en parte, con las gestiones de la universidad y con la disposición del sector productivo para contratar recién egresados de la UNAD en la ECACEN ZOCC.

\section{RESULTADOS Y DISCUSIÓN}

Al realizar el tratamiento y análisis de los datos mediante el uso del software PSPP, se pudo obtener una tabla con la cantidad de egresados por programa que respondieron la encuesta, tal como se observa a continuación:

Tabla 1: Cantidad de egresados que respondieron la encuesta. 


\begin{tabular}{|c|c|c|c|c|c|}
\hline Reveve de Wabr & valor & Frevernos & novastaje & fovantale valde & Paventaje Aantulats \\
\hline & Afreingraden de Empresas & $\mathbf{1 9 2}$ & 61,15 & $6 \mathrm{t}, 15$ & 61,15 \\
\hline & Especialuacein en Gerence Estrategca de Mrcades & 11 & 3,50 & 3,50 & 64,65 \\
\hline & Feecintaseien en Genteon de Prapectas & 47 & 24,97 & 14,97 & 70,62 \\
\hline & Moestrig en Adriristrachon de Orgavicaciones & 14 & 4,46 & 4,45 & 8409 \\
\hline & Teconologia an Cestion houpecieria & 4 & 1,27 & 2.27 & 6535 \\
\hline & 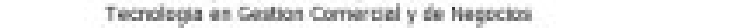 & 25 & 7,50 & 7,96 & 20,31 \\
\hline & Tecrolsgis en Gestion hostriel & 4 & 1.91 & L.9: & 95.22 \\
\hline & Teovalogis en Gesoun de Empresas Asoothes y Orgenizodioses Comunterias & 2 & , 64 & s4 & 9685 \\
\hline & 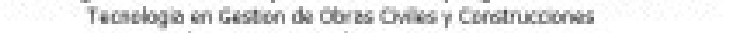 & 12 & 3,62 & 3,92 & 90,69 \\
\hline & Tecnolagia en Gestion de Tranipartes & a & 32 & 12 & 100.09 \\
\hline \multicolumn{2}{|r|}{$e_{2}$} & 314 & 160,0 & 106,4 & \\
\hline
\end{tabular}

Fuente: elaboración propia con el software PSPP.

De manera adicional se trataron los datos para determinar las opciones que la universidad brinda a los egresados para ubicarse, claro está, desde su rol de IES.

En primer lugar, se buscó determinar si los egresados piensan que el estudiar en la UNAD les ha permitido adquirir competencias suficientes para ingresar al mundo laboral en la profesión que eligieron en particular. Esto se evidencia en la siguiente tabla de contingencia lograda:

Tabla 2: Percepción de egresados frente a las competencias.

\begin{tabular}{|c|c|c|c|c|}
\hline \multirow{3}{*}{$\begin{array}{r}\text { Centro del cual es egresado el encuestado } \\
\text { Dosquebradas }\end{array}$} & Si el egresado pienso que el estudiar en lo UNAD ie & tes para ingreso & esion que elligio & \\
\hline & No obtuvo respuesta & No. & Si. & Total \\
\hline & $\begin{array}{r}4,00 \\
5,06 \% \\
\end{array}$ & $\begin{array}{r}10,00 \\
12,66 \%\end{array}$ & $\begin{array}{r}65,00 \\
82,28 \%\end{array}$ & $\begin{array}{r}79,00 \\
100,00 \%\end{array}$ \\
\hline La Dorada & , $00 \%$ & $\begin{array}{r}5,00 \\
14,71 \%\end{array}$ & $\begin{array}{r}29,00 \\
85,29 \%\end{array}$ & $\begin{array}{r}34,00 \\
100,00 \%\end{array}$ \\
\hline Medellin & 3,00 & 20,00 & 132,00 & 155,00 \\
\hline & $1,94 \%$ & $12,90 \%$ & $85,16 \%$ & $100,00 \%$ \\
\hline Quibdo & 1,00 & 3,00 & 17,00 & 21,00 \\
\hline Turbo & $4,76 \%$ & $\frac{14,29 \%}{300}$ & $80,95 \%$ & $100,00 \%$ \\
\hline Turoo & $.00 \%$ & $12,00 \%$ & $88,00 \%$ & $\begin{array}{r}25,00 \\
100,00 \%\end{array}$ \\
\hline Total & 8,00 & 41,00 & 265,00 & 314,00 \\
\hline
\end{tabular}

Fuente: elaboración propia con el software PSPP.

Se puede apreciar como más del $80 \%$ de los egresados de cada uno de los centros considera que la universidad le ayudó a desarrollar competencias para el mercado laboral.

De manera complementaria se revisó la opinión sobre la necesidad enseñar a los egresados el uso de bolsas de empleo para una mayor facilidad en la ubicación laboral, pero las respuestas dan cuenta de un bajo interés en el asunto; en este ítem el centro que marcó mayor importancia fue el correspondiente a los egresados del CEAD Medellín con un 53,19\% de respuestas afirmativas (figura 1 ).

Figura 1: Interés de los egresados sobre el uso de bolsas de empleo. 


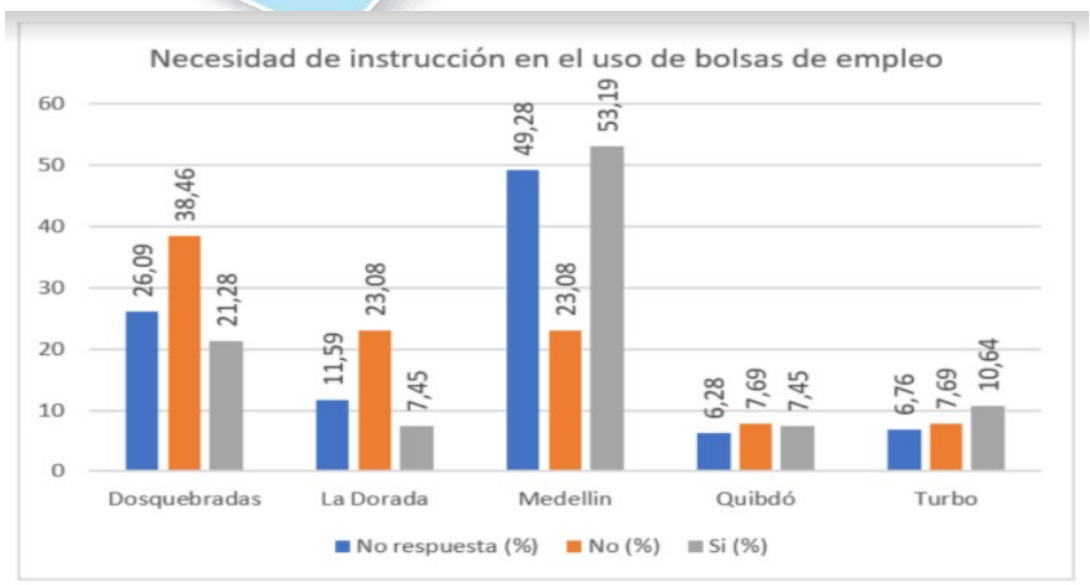

Fuente: elaboración propia.

Al verificar si los encuestados conocen las acciones de la UNAD para ayudar a la ubicación laboral de sus egresados, un gran porcentaje no respondió a la pregunta y en relación con el apoyo que los graduados perciben por parte de la oficina de egresados el comportamiento es muy similar.

Figura 2: Conocimiento acciones de la UNAD

¿Conoce de acciones de la UNAD para la ubicación laboral"

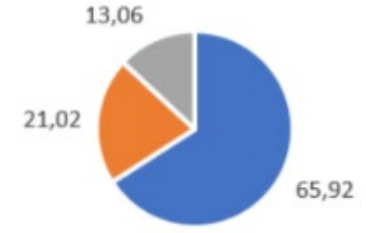

- No respuesta $(\%)=\mathrm{No}(\%)=\mathrm{Si}(\%)$
Figura 3: Acciones percibidas

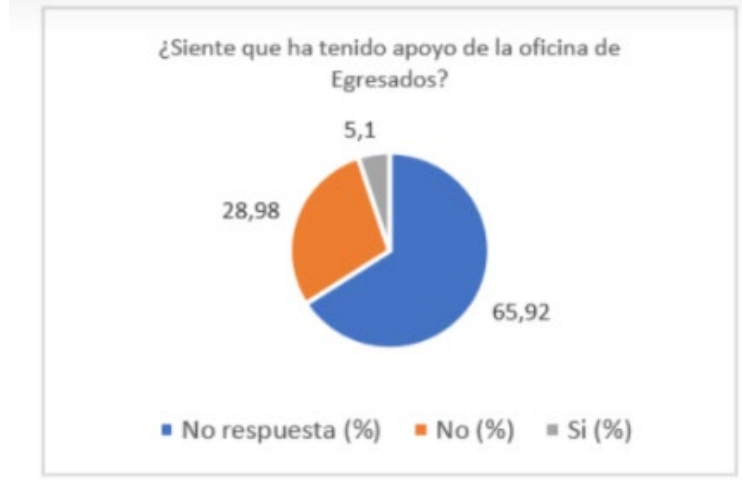

- No respuesta $(\%) \quad$ = No (\%) $=\mathrm{Si}(\%)$

Fuente: elaboración propia.

Fuente: elaboración propia.

Tabla 3: Importancia de la oficina de egresados.

\begin{tabular}{|c|c|c|c|c|c|c|}
\hline \multirow[b]{2}{*}{ Importanoia que los encuestados consisera que tiene la oficina de egresados para su ubicación laboral } & \multicolumn{5}{|c|}{ Centro del cual es egresado el encuestado } & \multirow[b]{2}{*}{ Total } \\
\hline & Dosquebradas & La Dorada & Medellin & Quibdo & Turbo & \\
\hline $\begin{array}{lll} & \text { Alta. }\end{array}$ & $\begin{array}{r}4,00 \\
5,06 \%\end{array}$ & $\begin{array}{r}3,00 \\
8,82 \%\end{array}$ & $\begin{array}{r}18,00 \\
11,61 \%\end{array}$ & $\begin{array}{r}1,00 \\
4,76 \%\end{array}$ & $\begin{array}{r}3,00 \\
12,00 \%\end{array}$ & $\begin{array}{r}29,00 \\
9,24 \%\end{array}$ \\
\hline Baja. & $\begin{array}{r}4,00 \\
5,06 \%\end{array}$ & $\begin{array}{r}3,00 \\
8,82 \%\end{array}$ & $\begin{array}{r}8,00 \\
5,16 \%\end{array}$ & $\begin{array}{r}3,00 \\
14,29 \%\end{array}$ & $\begin{array}{r}5,00 \\
20,00 \%\end{array}$ & $\begin{array}{r}23,00 \\
7,32 \%\end{array}$ \\
\hline Media. & $\begin{array}{r}6,00 \\
7,59 \%\end{array}$ & $\begin{array}{r}2,00 \\
5,88 \%\end{array}$ & $\begin{array}{r}19,00 \\
12,26 \%\end{array}$ & $\begin{array}{r}4,00 \\
19,05 \%\end{array}$ & $\begin{array}{r}1,00 \\
4,00 \%\end{array}$ & $\begin{array}{r}32,00 \\
10,19 \%\end{array}$ \\
\hline Muy alta. & $\begin{array}{r}7,00 \\
8,86 \%\end{array}$ & $\begin{array}{r}1,00 \\
2,94 \%\end{array}$ & $\begin{array}{r}7,00 \\
4,52 \%\end{array}$ & .00 & $\begin{array}{r}2,00 \\
8,00 \%\end{array}$ & $\begin{array}{r}17,00 \\
5,41 \%\end{array}$ \\
\hline No obtuvo respuesta & $\begin{array}{r}54,00 \\
68,35 \%\end{array}$ & $\begin{array}{r}24,00 \\
70,59 \%\end{array}$ & $\begin{array}{r}102,00 \\
65,81 \%\end{array}$ & $\begin{array}{r}13,00 \\
61,90 \%\end{array}$ & $\begin{array}{r}14,00 \\
56,00 \%\end{array}$ & $\begin{array}{r}207,00 \\
65,92 \%\end{array}$ \\
\hline No tiene importancia. & $\begin{array}{r}4,00 \\
5,06 \%\end{array}$ & $\begin{array}{r}1,00 \\
2,94 \%\end{array}$ & $\begin{array}{r}1,00 \\
, 65 \%\end{array}$ & $.00 \%$ &, $00 \%$ & $\begin{array}{r}6,00 \\
1,91 \%\end{array}$ \\
\hline Total & $\begin{array}{r}79,00 \\
100,00 \%\end{array}$ & $\begin{array}{r}34,00 \\
100,00 \%\end{array}$ & $\begin{array}{r}155,00 \\
100,00 \%\end{array}$ & $\begin{array}{r}21,00 \\
100,00 \%\end{array}$ & $\begin{array}{r}25,00 \\
100,00 \%\end{array}$ & $\begin{array}{r}314,00 \\
100,00 \%\end{array}$ \\
\hline
\end{tabular}

Fuente: elaboración propia con el software PSPP.

Tal como se puede observar, hay un alto porcentaje de egresados que no da respuesta a varias de las preguntas claves de la encuesta y esto puede ser 
un síntoma de la falta de conocimiento de los servicios que la UNAD tiene a su disposición, asunto que puede dar paso a nuevas investigaciones; también es apreciable la cantidad de egresados que no considera importante la necesidad de ser instruidos en la gestión de bolsas de empleo, esto da cabida a la posibilidad de que las personas no tengan muy claros todos los aspectos que se deben considerar a la hora de postularse a una vacante, dado que no solo se trata de entregar una hoja de vida sino de estructurarla adecuadamente con su experiencia, estudios y demás elementos que dan cuanta de un buen perfil profesional, el cual debe ser presentado solo a algunas vacantes seleccionadas de manera cuidadosa en vez de ser enviado indiscriminadamente porque esto último representaría la incapacidad de una persona para aspirar adecuadamente a vacantes en las cuales puede aplicar.

Por otro lado, es posible observar cómo más del $84 \%$ de los encuestados manifiesta que el estudiar en la UNAD le brindó competencias suficientes para ingresar al mundo laboral en la profesión que eligió, también resalta la alta participación de encuestados ya que se superó la cifra mínima requerida para la muestra requerida en esta investigación. Finalmente, se presenta la nube de palabras que arrojó el software Atlas.ti después de procesar la bibliografía suministrada para determinar los factores mas comunes frente al tema de este estudio

Figura 4: Nube de palabras sobre la relación de egresados y el empleo.

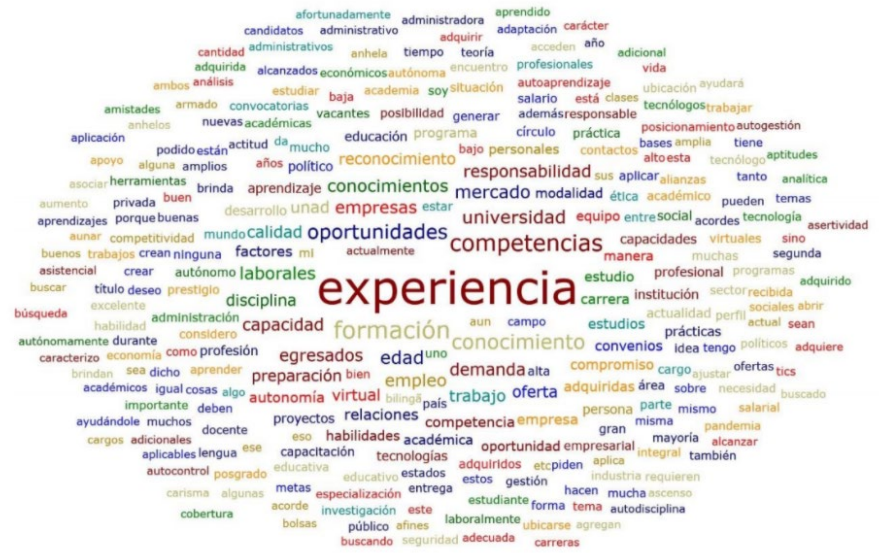

Fuente: elaboración propia con el software Atlas.ti.

A pesar de que en el estudio se logó importante información de carácter primario y secundario, es necesario mencionar la posibilidad de profundizar en un tema tan extenso y profundo en el cual, mediante el presente trabajo, solo se dio una mirada global de un tema tan complejo que amerita ser abordado con nuevos estudios más específicos a partir de los datos logrados hasta el momento. 


\section{CONCLUSIONES}

A pesar de que en la literatura consultada se menciona una gran variedad de factores que intervienen en la relación entre los egresados y el empleo, se encontró que los cinco más mencionados son: experiencia, formación, competencias, conocimiento y oportunidades.

La falta de respuesta de los egresados a varias preguntas claves de la encuesta permite pensar en el aparente desconocimiento de los servicios que la UNAD tiene a su disposición, esto constituye una gran oportunidad para desarrollar una estrategia de fortalecimiento de las relaciones entre ambas partes, de esta forma no solo los egresados podrán encontrar otras opciones de empleo sino que la universidad también puede llegar a mejores acercamientos con el sector productivo a través de estas gestiones.

Los resultados obtenidos de la consulta en fuentes bibliográficas permiten observar como el entorno demanda cada vez más capacidades, principalmente de tipo intelectual en las personas. En la actualidad los egresados universitarios no son ajenos a esta situación y, en ocasiones, enfrentan dificultades de acceso al mercado laboral que pueden ser resueltas mediante una adecuada articulación de las relaciones existentes entre las IES, los egresados y el sector productivo.

Una de las posibles formas de mejorar las oportunidades de acceso al mercado laboral de los egresados de la ECACEN ZOCC corresponde a la creación una red que articule y dinamice la relación entre los egresados, la UNAD y el sector productivo.

\section{REFERECIAS LABORALES}

Arall, J. J., \& Molías, L. M. (2019). Aspectos de la competencia digital para la empleabilidad. REOP - Revista Española de Orientación y Psicopedagogía, 30(2), 67-84. https://doi.org/10.5944/reop.vol.30.num.2.2019.25339

Banco de la Republica. (2019). Resultados de búsqueda. https://totoro.banrep.gov.co/estadisticaseconomicas/faces/pages/charts/line.xhtml?facesRedirect=true

DANE. (2018). ¿Cuántos somos?

https://www.dane.gov.co/index.php/estadisticas-portema/demografia-y-poblacion/censo-nacional-de-poblacion-y-vivenda2018/cuantos-somos

Fern\&aacute, A. R., ndez, \& Gavilanes, J. A. (2015). Diagnostico de la insercion laboral de graduados de administracion de empresas de La Universidad Regional Autonoma de los Andes. Pedagog\&iacute; a 
Universitaria, 20(2), 58-77.

https://go.gale.com/ps/i.do?p=AONE\&sw =w\&issn $=16094808 \& v=2.1 \& i$

$\mathrm{t}=\mathrm{r} \& \mathrm{id}=\mathrm{GALE} \% 7 C A 466782816 \&$ sid $=$ googleScholar\&linkaccess $=$ abs

Fischer, S., Dornbusch, R., \& Startz, R. (1985). Macroeconomia. McGraw Hill.

González Jaimes, E. I., \& Miguel Silva, Ma. G. (2017). Egresados universitarios y sus pertinencias para el ingreso a la empleabilidad o la auto empleabilidad. https://core.ac.uk/download/pdf/154795648.pdf

Morales, L. F., \& Medina, D. (2019). Fluidez del mercado laboral y resultados en materia de empleo en Colombia: Evidencia derivada de datos enlazados de empleadores y empleados. Revista de la CEPAL, 2019(127), 123-148. https://doi.org/10.18356/b6cf5907-es

Nina, A., \& Eliana, N. (2019). Desajuste educativo en el primer empleo de los jóvenes egresados universitarios. Pontificia Universidad Católica del Perú.

http://tesis.pucp.edu.pe/repositorio/handle/20.500.12404/15146

Romero Fernández, A., \& Álvarez Gavilanes, J. (2015). DIAGNÓSTICO DE LA INSERCIÓN LABORAL DE GRADUADOS DE ADMINISTRACIÓN DE EMPRESAS DE LA UNIVERSIDAD REGIONAL AUTÓNOMA DE LOS ANDES. DIAGNOSIS TO EMPLOYMENT OF GRADUATES OF BUSINESS ADMINISTRATION OF THE UNIVERSIDAD REGIONAL AUTÓNOMA DE LOS ANDES., 20(2), 58-76. Fuente Académica.

https://bibliotecavirtual.unad.edu.co/login?url=http://search.ebscohos t.com/login aspx?direct $=$ true $\& d b=z b h \& A N=110040960 \& l a n g=e s \&$ site =eds-live\&scope $=$ site

Rus, T. I., \& Gragmena, A. J. F. (2018). Empleabilidad y expectativa de logro en la inserción laboral de los estudiantes universitarios. REOP - Revista Española de Orientación y Psicopedagogía, 29(2), 29-40. https://doi.org/10.5944/reop.vol.29.num.2.2018.23151 